\title{
The ongoing HIV epidemic in American youth: challenges and opportunities
}

\author{
Lao-Tzu Allan-Blitz ${ }^{1,2}$, Leandro A. Mena ${ }^{3}$, Kenneth H. Mayer ${ }^{4,5,6}$ \\ ${ }^{1}$ Department of Medicine, Division of Global Health Equity, Brigham and Women's Hospital, Boston, MA, USA; ${ }^{2}$ Department of Medicine, Boston \\ Children's Hospital, Boston, MA, USA; ${ }^{3}$ Department of Population Health Science, University of Mississippi Medical Center, Jackson, MS, USA; \\ ${ }^{4}$ Fenway Health, Boston, MA, USA; ${ }^{5}$ Division of Infectious Diseases, Department of Medicine, Beth Israel Deaconess Medical Center, Boston, MA, \\ USA; ${ }^{6}$ Harvard Medical School, Boston, MA, USA \\ Contributions: (I) Conception and design: All authors; (II) Administrative support: None; (III) Provision of study materials or patients: None; (IV) \\ Collection and assembly of data: All authors; (V) Data analysis and interpretation: None; (VI) Manuscript writing: All authors; (VII) Final approval of \\ manuscript: All authors. \\ Correspondence to: Lao-Tzu Allan-Blitz, MD. Brigham and Women's Hospital, 75 Francis Street, Boston, MA 02115, USA. \\ Email: lallan-blitz@partners.org.
}

\begin{abstract}
The incidence of human immunodeficiency virus (HIV) infection has been decreasing in the United States overall, except among youth, and in particular among Black and Latinx young men who have sex with men (MSM). In this review we summarize key drivers of the HIV epidemic among youth, as well as novel interventions geared specifically towards combating the epidemic among high-risk populations. Many factors driving the HIV epidemic among youth are related to systemic inequities, including lack of access to healthcare, inadequate education, and internalized and experience homophobia and racism. Developmentally, youth may feel that they are invulnerable and be willing to engage in risks. Moreover, HIV is often invisible for youth given advances in treatment and community stigma, limiting open discussion of risk and new preventive modalities. Outcomes from the HIV treatment cascade suggest that youth are less likely to be aware of their HIV infection status, less likely to link to and be engaged in care, and less likely to be virologically suppressed than older MSM and other populations of people living with HIV. Importantly, pre-exposure prophylaxis (PrEP) has been shown to be an effective tool for prevention of HIV infection that also appears to have disproportionately poor uptake among youth. Barriers to PrEP utilization appear to be quite heterogeneous, and include patient-, provider-, and structural-level barriers. Interventions important in improving HIV prevention will thus have to be multipronged and developed for culturally diverse populations. Cognitive behavioral therapy-based interventions are promising strategies as they are able to address a diverse array of barriers. New formulations of PrEP will also likely be instrumental in improving adherence. Since youth spend considerable amounts of time accessing digital media, the deployment of apps and other mobile phone-based interfaces offer unique opportunities to increase education and to facilitate HIV prevention for at risk youth. Multiple studies are underway to better inform the optimal delivery of treatment and prevention services for this complex and diverse population, and include novel sociobiological interventions and new modes of medication delivery that may lend themselves to overcoming obstacles specific to youth.
\end{abstract}

Keywords: Human immunodeficiency virus (HIV); youth; United States; MSM; pre-exposure prophylaxis (PrEP)

Received: 17 January 2020; Accepted: 05 June 2020; Published: 20 April 2021.

doi: $10.21037 /$ mhealth-20-42

View this article at: http://dx.doi.org/10.21037/mhealth-20-42 


\section{Introduction}

Modern antiretroviral treatment is highly effective in controlling human immunodeficiency virus (HIV) infection, preventing morbidity and mortality, and rendering virally suppressed persons non-transmittable, and pre-exposure prophylaxis $(\mathrm{PrEP})$ is effective in protecting people not infected with HIV when used as directed. However, there remains significant transmission of HIV infection in the United States, particularly among youth.

Factors driving that disproportionate spread of HIV infection include structural issues such as poverty, racism, homophobia, and youth-specific cultural issues, such as their perceptions of invulnerability and lack of familiarity with HIV. Biological issues, as well, range from the efficiency of anal intercourse for HIV transmission to challenges in impulse control and planful thinking in the immature brain. In this narrative review we describe the epidemiology of HIV infection, PrEP use, and risk behavior among youth in the United States, and discuss the rationale for new and innovative targeted biobehavioral interventions that may facilitate enhanced uptake of preventive strategies and improved HIV medication adherence among at risk youth, who will continue to bear an increasing burden of HIV infection, unless new and effective approaches are brought to scale.

\section{Epidemiology of human immunodeficiency virus (HIV) infection}

In recent years, there have been nearly 40,000 new diagnoses of HIV infection in the United States (1), and 1.7 million new cases worldwide (2). Among many factors likely driving continued transmission in the United States, more than $13 \%$ of the 1.2 million people living with HIV are unware of their infection (3). Furthermore, only $50 \%$ of those known to be infected are receiving effective treatment (3), thus there remains a large population of individuals who are infectious and capable of transmitting the virus to uninfected partners.

In the United States, continued HIV transmission seems to be of particular concern among minority youth. While the overall incidence of HIV infection has been stable or decreasing in most populations, the number of new HIV infections has increased among youth (Figures 1,2), particularly among Black and Latinx MSM, who bear the greatest burden of new infections (Figure 3) $(4,5)$. In 2017, 21\% of new HIV infection diagnoses were among individuals between the ages of 13 to 24 years old, and were primarily (87\%) among men (6). Among males, 93\% reported male-to-male sexual contact as their mode of HIV infection, whereas $86 \%$ of females reported heterosexual contact (6). The prevalence of HIV infection increased with age, with $21 \%$ of cases in youth being diagnosed in those between 15 to 19 years, while the rest were between 20 to 24 years old (6). Furthermore, youth were less likely to be aware of their HIV infection status, with roughly $40 \%$ of those infected with HIV aware of the infection compared to the national estimate of over $85 \%$ of older individuals (7). Of new HIV infection diagnoses in 2017, 51\% were among Black youth, and $25 \%$ were among Latinx individuals (6). The number of new HIV infection diagnoses reported in the United States was highest in the South. The increasing concentration of HIV in the South may reflect social and structural issues, such as decreased social mobility associated with poverty, limited access to health care because of a lack of health insurance, and lower average levels of education and health literacy (8). Among youth living with HIV in the South, $13 \%$ were economically dependent on a sex partner (8), which has been associated with increased sexual risk taking behaviors.

Behaviors associated with HIV acquisition among youth in the United States are similar to older populations, with the highest burden of HIV infection being among MSM and transgender women who engage in anal intercourse, and, to a lesser extent, people who share needles, and sex workers (7). It has been hard to elucidate specific HIV risk factors for young cisgender women, other than them having condomless sex with a male partner living with HIV. Because of the low rates of serostatus awareness and disclosure by some partners, it has been challenging to identify specific preventive guidance beyond knowing the HIV status of partners, and if infected, whether they are virologically suppressed. Of note, pregnancy and the post-partum period appear to increase the risk for $\mathrm{HIV}$ infection (8).

Youth in general are at particularly increased risk for HIV because of biologic and physical factors that occur uniquely during adolescence. The young brain is not fully mature until the mid-20's, which may partially explain impulsiveness and lack of planning (6). Many youth yearn for increasing autonomy from parents and caregivers, while placing increased importance on peer relationships resulting in enhanced vulnerability to peer influence. Those developmental issues may lead to increased risktaking behavior as a part of experimentation, identity formation, and often in response to peer pressure, with 


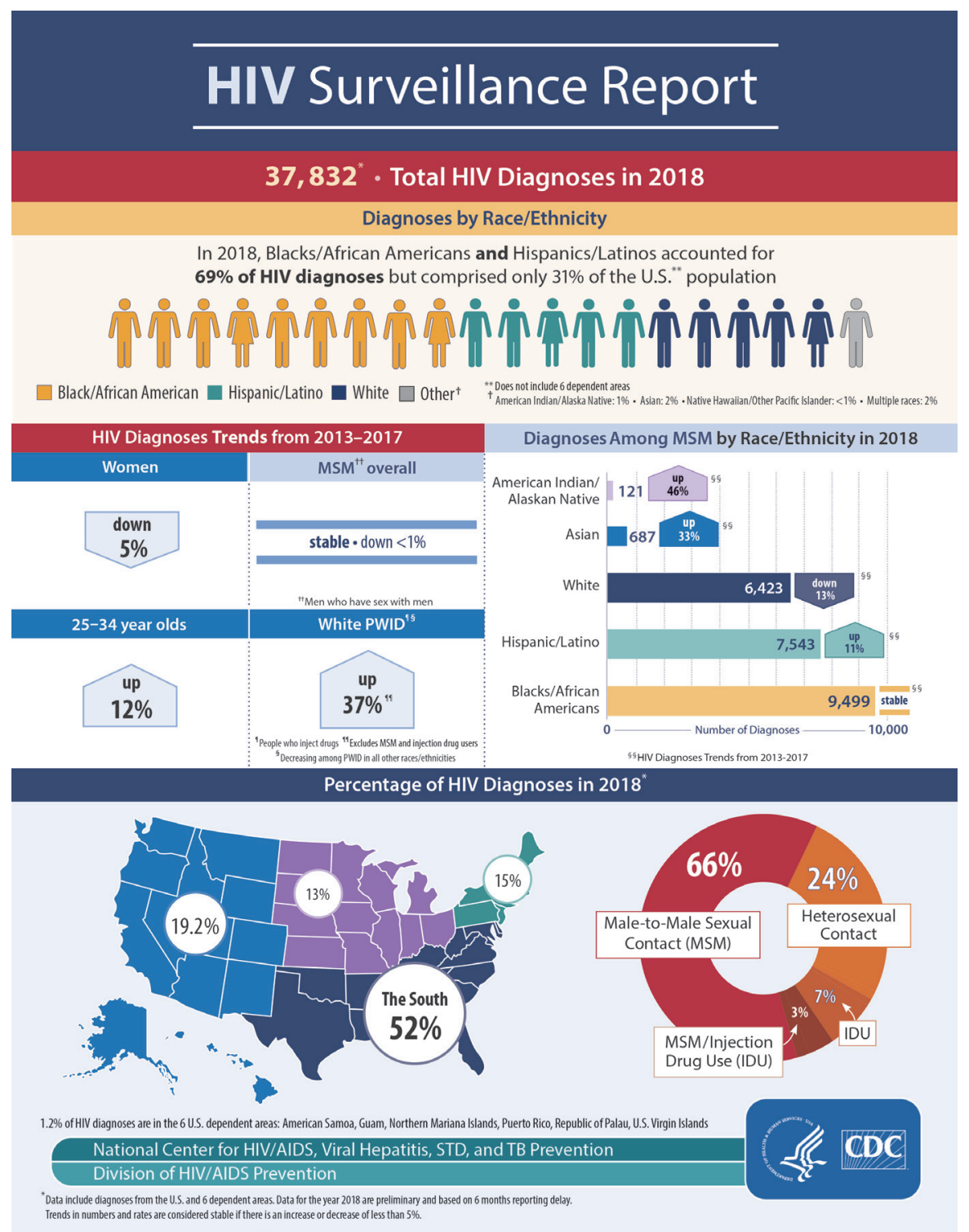

Figure 1 Center for Disease Control and Prevention 2018 HIV Surveillance Report indicating trends in HIV infection among different populations in the United States. HIV, human immunodeficiency virus.

minimal support structures (9). Stigma surrounding HIV infection, in particular, seems to be highly prevalent among youth in the United States, most notably in Southern states compared to Northeastern states (10), where community education and stigma reduction programs may be less prevalent, potentially limiting healthcare seeking behavior even further.

Black and Latinx young MSM appear to be at a disproportionate risk for HIV infection (6). The engagement of racial and ethnic minority youth with the healthcare system is complex. Although young Black MSM in Chicago reported less high-risk sexual behavior and more HIV testing than young White MSM, they were less likely to achieve viral suppression after diagnoses, reflecting alienation from healthcare services perceived to be culturally insensitive (11). Network analyses, however, identified more homogeneity and higher rates of concurrent sex partners among young Black MSM, which suggests that the social network, rather than individual factors, may be a primary driver of the racial disparities in HIV infection among youth 


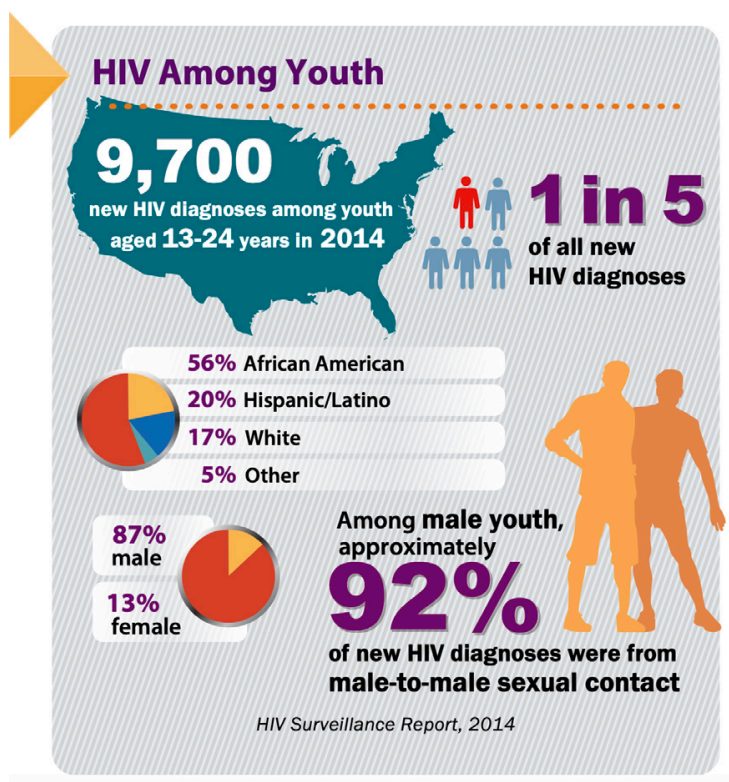

Figure 2 Data from the Center for Disease Control and Prevention 2014 HIV Surveillance Report demonstrating characteristics of youth in the United States who are living with HIV infection. HIV, human immunodeficiency virus.

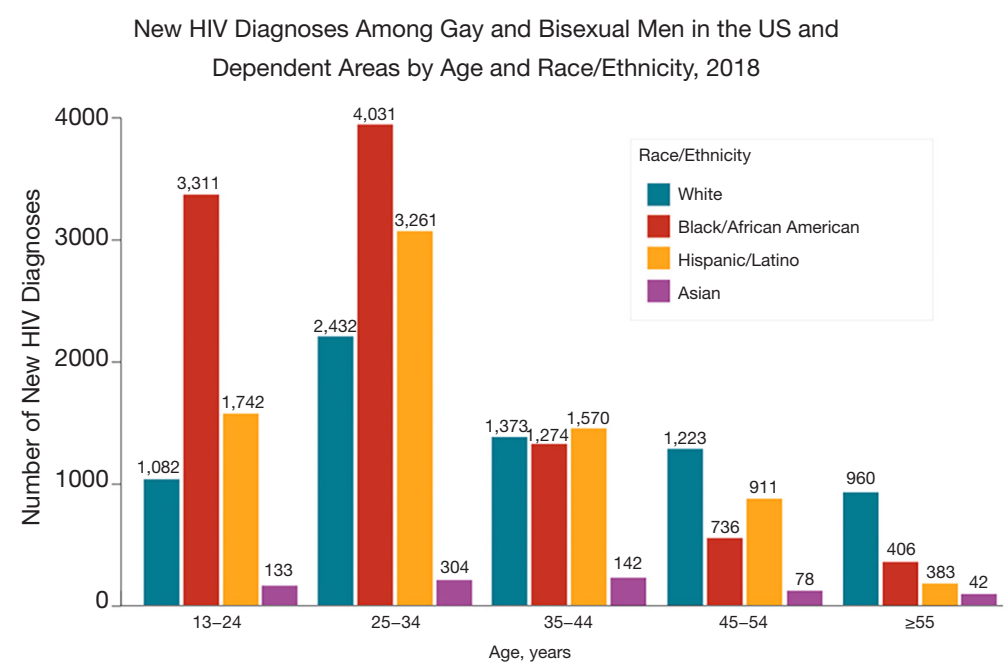

Figure 3 Prevalence of new diagnoses of HIV infection among men who have sex with men in the United States. HIV, human immunodeficiency virus.

$(11,12)$. That pattern of behavior is known as assortative mixing, in which individuals are more likely to choose sex partners from within their own racial or ethnic group. That tends to concentrate HIV infection within subgroups, since HIV prevalence in the limited partner pool tends to be higher than among the larger population of non-minority MSM. Similar logic holds for network factors driving increasing rates of HIV infection among youth overall, as such youth-focused networks may be more homogenous than their adult counterparts with HIV uninfected youth being more likely to encounter young partners who are not virally suppressed, compared to older individuals with HIV.

\section{The HIV care continuum in youth}

The HIV prevention and care continua are the cascades of 
Table 1 Factors associated with reduced antiretroviral therapy uptake among youth linked to HIV care in the United States

Patient-level barriers
Advanced disease
Asymptomatic infection
Substance use
Frustration/dissatisfaction with the health system
Fear or stigma
Concomitant psychiatric disorders
Socioeconomic barriers
Limited health insurance
Insufficient youth-friendly services
Structured racism
Lack of social support
Housing insecurity
Transportation challenges
Medication related barriers
Complex therapy regimen

HIV, human immunodeficiency virus.

care that include bio-behavioral prevention strategies such as screening, risk-behavior counselling, and PrEP uptake, as well as linkage to antiretroviral therapy and followup care for HIV infection $(13,14)$. Youth face challenges at every step of the HIV care continuum. Awareness of HIV infection status is significantly less among youth compared to adults (15). Using HIV testing as a surrogate for awareness of HIV infection, the Centers for Disease Control and Prevention found that HIV testing increased among youth with each increasing educational grade, among those who reported being educated about HIV and AIDS, and those reporting younger age at first sexual encounter (16). HIV testing may be more prevalent among young women compared to young men, and among young Black and Latinx populations $(16,17)$. Other factors associated with increased HIV testing include a history of having a sexually transmitted infection, having three or more sexual partners in the preceding three months, inconsistent condom use, identifying as an MSM or female who has had sex with an MSM, substance use, or reporting sex with a partner known to be living with HIV infection or of unknown infection status $(18,19)$. Furthermore, youth using Medicaid, those with a primary care physician, and/ or those who had received healthcare in the past year were more likely to have been tested for HIV (20).

However, once linked to care, initiation of antiretroviral therapy may be limited by several factors including unstable housing or substance abuse (21). Even after initiation, adherence to therapy can be a significant challenge for youth for a myriad of reasons (22-28). See Table 1 for a summary of factors associated with reduced antiretroviral therapy use among youth connected to HIV care in the United States.

Retention in HIV care is also a challenge for youth beyond adherence to antiretroviral medications. Youth are less likely to be retained in care compared to adults $(29,30)$. Lack of services for medical gender affirmation and experienced stigma in HIV care were independently associated with increased odds of missing an HIV care appointment in a study among young transgender women in the United States (31).

Further complicating the matter, viral resistanceeither transmitted or developed as a consequence of poor adherence, drug interactions, malabsorption, or other causes-may result in poor viral suppression. The prevalence of transmitted HIV infection with resistance to first-line therapy among youth range from $9 \%$ to $18 \%$ in recent studies $(32,33)$.

Thus, for diverse reasons youth are less likely to know their HIV infection status, be linked to or retained in care, adhere to antiretroviral therapy regimens, and to achieve and maintain viral suppression. Overall, estimates suggest that between $29 \%$ to $73 \%$ of youth aware of being infected with HIV engage in medical care within 12 months of diagnosis (15). Furthermore, only $54 \%$ of youth initiated on antiretroviral therapy achieve viral suppression, while more than half are not retained in care (15). In total, less than $10 \%$ of youth living with HIV in the United States achieve and maintain viral suppression (15).

\section{Pre-exposure prophylaxis use and high-risk youth}

PrEP using a combination once daily pill of tenofovir disoproxil fumarate (TDF) or tenofovir alafenamide (TAF) and emtricitabine (FTC) is an effective tool for the prevention of HIV infection, and when taken consistently, prevents more than $95 \%$ of infections $(34,35)$. The effectiveness of PrEP, however, is highly dependent on medication adherence. Drug levels consistent with participants taking four doses of TDF/FTC per week 
Table 2 Factors associated with reduced PrEP uptake among youth in the United States

Patient-level barriers
Lack of awareness
Lack of access to healthcare
Stigma
Poverty
Concerns about side effects or drug-drug interactions
Concerns about proper storage of medication
Lack of social support
Low perceived risk of HIV infection
Burden of daily-dosing
Burden of frequent clinic visits and lab testing
Structural barriers
Limited health insurance
Insufficient youth-friendly services
Stigma
Structural racism
Provider-level barriers

PrEP, pre-exposure prophylaxis.

have been associated with a $96 \%$ reduction in risk for HIV infection for cisgender men and transgender women who have sex with men, while levels consistent with daily use were associated with greater than $99 \%$ risk reduction for all populations; conversely, taking fewer than four doses per week may not provide adequate protection (34). Medication adherence is a particular challenge among young MSM (36). Furthermore, young MSM are more likely to engage in high-risk sexual behavior than older MSM (37), and are therefore at higher risk for HIV infection (38), suggesting their need for PrEP is greater than older MSM.

While PrEP uptake in the United States has increased in recent years, the uptake has been slowest among those younger than 24 years old (39). PrEP use in the United States among youth is lowest in the South and highest in the Northeast (39), a discrepancy which is likely driven by related disparities of healthcare financing, health literacy, stigma-all similar drivers of HIV infection among the same population. Youth are more likely to report a low perceived risk for HIV infection (40), and thus may be less inclined to take PrEP, and, once started, younger men are more likely to discontinue PrEP (41). There are numerous barriers that drive such low PrEP uptake among youth (Table 2), which must be overcome in order to combat the HIV epidemic.

Patient-level barriers among youth include limited familiarity with antiretroviral medications, concerns about product storage, and lack of social support (38). Stigma is of particular concern as there may be individual as well as structural factors driving such perceptions. And beyond experienced stigma, anticipatory stigma may deter healthcare seeking in the first place. Other structural barriers include: limited health insurance and insufficient access to youth-friendly services (38). And finally, there are provider-level barriers. Although US-based clinicians specializing in Adolescent Medicine generally reported that prescribing PrEP in accordance with the 2012 Centers for Disease Control and Prevention guidelines was compatible with their practice (42), several prescribers preferred to prescribe to those 18 years of age or older because of their perceptions of ethical and legal issues when prescribing to a minor (42). The laws regarding the ability of adolescents to consent for treatment and prevention of HIV infection differ by state (43), thus further complicating prescribers ability to offer PrEP.

Specific populations with the lowest PrEP uptake include young Black and Latinx MSM, women (cisand transgender), and people who inject drugs $(8,44)$. Drivers of such disparities are multifactorial and often overlap. Lack of awareness about PrEP, particularly among Black MSM, was shown to be related to lack of access to healthcare. In general, young Black MSM with some access to healthcare (e.g., prior testing for sexually transmitted infections or with a documented primary care provider) were more likely to be aware of PrEP than those who had not been tested or who had not seen a primary care provider (45). Black MSM are also more likely than other races to report PrEP related stigma (46). Perceived racism and medical mistrust also contribute suboptimal PrEP uptake, particularly among racial and ethnic minority MSM (47). Among young Latinx MSM, a qualitative study noted specific barriers to PrEP including perceived burden of daily dosing, concern regarding risks associated with PrEP use as well as side effects (specifically renal injury and decreased bone density), stigma in their social environment, and finally provider influences discouraging PrEP use (48).

Another barrier to understanding PrEP uptake in young 
MSM has been the limited number of research studies specifically focused on youth (49). There have been two PrEP safety and acceptability trials among youth in the United States $(44,50)$. The Adolescent Trials Network (ATN) study 110 enrolled youth 18 to 24 and ATN 113 enrolled youth 15-17, but were essentially the same protocol, which evaluated PrEP adherence among youth randomized to an individualized versus a group behavioral intervention. Although both studies demonstrated safety and overall PrEP acceptability, over the course of 6 months, the majority of youth did not maintain protective PrEP levels and HIV incidence remained high. Interestingly, a large number of the youth had drug levels consistent with taking 2 to 4 pills a week. Such infrequency of dosing does not meet standards for sufficient HIV prevention, but seems to reflect an attempt to comply with the dosing regimen. Notably, both studies also documented a decline in adherence after three months, once the follow-up visits went from monthly to quarterly visits. That finding suggests that more frequent visit schedules for youth may be a strategy for improving adherence, which deserves further study. Similar findings have been reported in preliminary data from other ongoing international studies (51). A recent study confirmed the capacity of young Black MSM to selfconsent to research, thus supporting youth autonomy, since requiring parental consent may inhibit youth's willingness to access PrEP and/or participate in prevention studies (52).

Young transgender populations constitute another subpopulation at high risk for HIV infection who may benefit from, but who have notably poor uptake of PrEP (53). Again, a paucity of data limits our understanding of the perceptions about PrEP use and barriers to PrEP uptake among that population. Part of the reason for such limited data is the lack of participation from transgender youth in PrEP and HIV prevention trials, which may be related to a reluctance to discuss gender identity with study staff, the need for guardian consent, concerns over medication side effects, perceived burden of care (e.g., daily medication dosing and quarterly meetings), and lack of concern about HIV infection (54). Primary barriers to PrEP use among transgender and nonbinary youth in the United States include medication cost and concerns about drug interaction with hormone therapy (55), as well as fear of stigma (56). In one study, young male and transgender female sex workers in Puerto Rico expressed an interest in oral PrEP, but reported concerns about side effects as a primary barrier to use (57). Low awareness of PrEP was another barrier in a multi-center study of young transgender women in the
United States, which found that only 5\% of PrEP-eligible individuals reported ever taking PrEP (58). Similar barriers have been reported among transgender adults from other countries $(59,60)$.

Data from the United States are limited among youth who inject drugs. One study from Canada reported that younger age was associated with an increased willingness to use PrEP among people who inject drugs (61). Overall, however, willingness to use PrEP among people who use drugs may not be as high as in other high-risk groups, and is reportedly contingent upon demonstrable efficacy in preventing HV infection (62). Notably, there have been trials demonstrating the benefits for PrEP use among people who inject drugs, though not specifically among youth who inject drugs in the United States (63). Primary barriers to PrEP use among people who inject drugs are similar to other high-risk groups, and include concerns about cost, frequency of dosing, condom use, and regular blood draws, clinic visits, and HIV testing (62). Integrating PrEP into substance abuse treatment settings, such as methadone clinics, is another promising strategy to overcome access barriers among individuals either currently on or seeking treatment for drug abuse (64), though acceptability specifically among youth remains to be studied.

Another important population for whom PrEP is underutilized is at risk young women. Challenges have included the identification of the young women who could benefit most from PrEP, given the challenges in ascertaining partners' risks. Additionally, the bar for adherence appears to be higher for women, requiring stricter adherence to daily pill use. Data generated from studies that enrolled women 18 years of age and older have suggested that higher adherence is necessary to establish effective drug levels of tenofovir and its active intracellular metabolite in cervical tissues compared to rectal tissues $(65,66)$. Similarly, the time it takes to achieve protective levels in cervical and vaginal tissue may be significantly longer than in rectal mucosa. Thus, adherence is particularly important in young women, especially considering the potential for disinhibited sexual risk behavior among those taking PrEP (67). In addition, gender-related dynamics may complicate HIV prevention for young women. For example, one study noted that women who report an inability or reluctance to negotiate condom use may be more likely to experience partner violence (68). Thus, among young women, while PrEP may be intended as a tool for HIV prevention, issues like the potential for disinhibited sexual risk behavior, the heightened dependence on adherence, and how partners 
react, could paradoxically increase the risks for HIV acquisition.

\section{Novel strategies to improve HIV prevention among youth}

There are limited data from trials evaluating interventions to improve PrEP adherence among youth. More frequent visits, as mentioned above, may be one approach unique to adolescents. Other interventions which may specifically improve PrEP use among youth include mobile and webbased applications, gamification, social interventions including relationship skill building and cognitive behavioral therapy-based interventions, as well as home PrEP delivery systems $(69,70)$, all of which are under study in the University of North Carolina and Emory Center for Innovative Technology (iTECH) consortium. The iTECH consortium specifically aims to reduce the burden of HIV infection via the development and implementation of novel treatment and prevention strategies utilizing technology-based interventions among youth (71). One such intervention utilized a computer simulation model to evaluate the effectiveness of ongoing and forthcoming ATN interventions (72). The use of online and social media platforms as a medium for HIV and PrEP related interventions is also a vital component of novel strategies particularly among youth; such interventions facilitate content tailored to individual needs on a scale otherwise not feasible for in-person visits (73).

Given the unique, heterogeneous, and culturally complex framework limiting the HIV prevention continuum among youth in the United States, a holistic approach is warranted. A study among racially diverse minority (predominantly Black and Latinx) young MSM, demonstrated feasibility and acceptability of enrollment and retention in a PrEP study when the intervention was combined with a groupbased behavioral intervention that was culturally-tailored to address HIV risk and the social stigmas experienced by being someone who is both a racial and sexual minority person (74). A separate cognitive behavioral therapybased intervention originally developed for antiretroviral medication adherence was shown to be beneficial in improving PrEP adherence among adolescents in a pilot study (75), and is now the focus of an ongoing study using those same methods coupled with SMS reminders (76).

SMS-based interventions themselves may be an important intervention in isolation or coupled with other interventions among youth, given the recurrent findings of waning adherence with less frequent clinic visits. Such reminders have been shown to augment short term behavior (77), and antiretroviral medication adherence $(78,79)$.

Finally, reducing the burden of care that is associated with PrEP may target specific barriers identified among young high-risk populations. One ongoing study is evaluating the utility of a home-care system for PrEP which will obviate the need to travel to a clinician's office or laboratory for testing by incorporating at-home specimen collection and behavioral surveillance (80). Similarly, new formulations of PrEP which require a less rigid dosing regimen will likely improve adherence.

\section{Future formulations of PrEP in the Pipeline}

Important to any discussion of new formulations of PrEP is the preface that demonstrating similar effectiveness will be a challenge for clinical trials in the era of effective control and prevention strategies. That being said, there are several new formulations in the pipeline. However, any new formulation of PrEP alone will not be successful unless underlying youth-specific social, structural and behavioral issues are not fully addressed.

The United States Food and Drug Administration recently approved the use of Tenofovir alafenamide (TAF)/ FTC combination pill (81). TAF is similar to TDF and has been shown to have less of an impact on renal and bone function, and can be administered at a lower dose (i.e., a smaller pill). A recent study comparing the preventative efficacy of TAF/FTC and TDF/FTC among MSM and transgender women demonstrated non-inferiority of TAF/ FTC (82). TAF/FTC may directly address youth's concerns over side effects, however will likely still require daily dosing, until studies of alternative dosing regimens are completed.

There are efficacy trials underway for injectable, longacting cabotegravir (an integrase strand transfer inhibitor) which can be dosed every eight weeks, and has previously been shown to be safe and well tolerated (83). Those ongoing studies include joint protocols sponsored by the ATN and the HIV Prevention Trials Network.

Similarly, there are ongoing trials evaluating implanted or transdermal antiretroviral medications that can be dosed every few months $(84,85)$. Such formulations of PrEP may show much higher rates of adherence, particularly among youth, however, other logistic challenges will need to be addressed, such as ensuring that youth remember when they need to come to clinic for infrequent dose administration. 
Other creative modes of delivery, including home visits, may be warranted, but will have to address concerns about stigma if they are to be successful. Furthermore, the risk of induced drug-resistant HIV infection is worth noting for any long-acting form of PrEP; as circulating drug levels fall below the threshold for protection upon discontinuation, a new exposure at that time may result in a resistant strain of the virus. Such a phenomenon is akin to what was noted in the PrEP efficacy trials among cases of PrEP initiation during acute HIV infection (86).

An intravaginal ring with dapivirine (a non-nucleoside reverse-transcriptase inhibitor) decreased the incidence of HIV infection in African women compared to placebo (87), and may specifically address the concerns over achieving optimally effective drug levels in cervical and vaginal mucosa with oral daily tenofovir-based regimens. Importantly, however, while studies have demonstrated a significant reduction in the incidence of HIV infection with consistent use off the dapivirine intravaginal ring, the level of protection was notably less than with oral PrEP (88).

Another preventive approach is immunoprophylaxis utilizing broadly neutralizing monoclonal antibodies (bnAb). Animal studies have been promising, and now efficacy trials are underway in humans evaluating one such antibody, VRCO1, which has been shown to be safe and well tolerated (89), while other bnAb with broader ranges of neutralizing activity are in development (90). Future studies will be necessary to evaluate optimal dosing regimens and while considering the challenges of maintaining adherence among youth. In future studies evaluating bnAbs or any other iteration of PrEP, explicit data among youth will be important for practical implementation to achieve meaningful preventive effectiveness.

\section{Conclusions}

Although effective treatment and prevention strategies for HIV infection have been developed, youth in the United States are still burdened with disproportionately high prevalence and incidence of infection. The uptake of prevention strategies, most notably PrEP, is also poorest among youth. Such disparities predominantly impact racial, ethnic, sexual and gender minority groups, and are driven by a heterogeneous and culturally complex set of factors, comparable to the diversity of youth populations in the United States. While new formulations of PrEP are in the pipeline and may address some youth-specific obstacles, much more work is needed to address the structural factors that contribute to the perpetuation of HIV among those populations.

\section{Acknowledgments}

Funding: The work is partially supported by the Biobehavioral and Community Science Core of the Harvard Center for AIDS Research.

\section{Footnote}

Provenance and Peer Review: This article was commissioned by the Guest Editor (Lisa Hightow-Weidman) for the series "Technology-based Interventions in HIV Prevention and Care Continuum among American Youth" published in mHealth. The article has undergone external peer review.

Conflicts of Interest: All authors have completed the ICMJE uniform disclosure form (available at http://dx.doi. org/10.21037/mhealth-20-42). The series "Technology-based Interventions in HIV Prevention and Care Continuum among American Youth" was commissioned by the editorial office without any funding or sponsorship. LM has received grants and personal fees from Gilead Science, ViiV Healthcare, from Merck, and Roche Molecular, grants from Binx Health, Evofem Inc., Click Diagnostics, Janssen Pharmaceutical, Prosoft Clinical, GSK, and SpeedDx Pty Ltd, outside the submitted work; KM has received unrestricted research grants to study antiretrovirals for prevention from Gilead Science, Merck, and ViiV Healthcare, and HIV vaccines with Janssen, outside the submitted work. The authors have no other conflicts of interest to declare.

Ethical Statement: The authors are accountable for all aspects of the work in ensuring that questions related to the accuracy or integrity of any part of the work are appropriately investigated and resolved.

Open Access Statement: This is an Open Access article distributed in accordance with the Creative Commons Attribution-NonCommercial-NoDerivs 4.0 International License (CC BY-NC-ND 4.0), which permits the noncommercial replication and distribution of the article with the strict proviso that no changes or edits are made and the original work is properly cited (including links to both the formal publication through the relevant DOI and the license). See: https://creativecommons.org/licenses/by-nc-nd/4.0/. 


\section{References}

1. Centers for Disease Control and Prevention. HIV Surveillance Report, 2016; vol 28. HIVSurveillance Report: Diagnoses of HIV Infection in the United States and Dependent Areas,2016. Centers for Disease Control and Prevention website. Available online: http://www.cdc. gov/hiv/library/reports/hiv-surveillance.html. Published November 2017. Accessed August 7th 2019.

2. World Health Organization. HIV/AIDS Data and Statistics. Updated 2019. Available online: https://www. who.int/hiv/data/en/. Accessed August 7th, 2018.

3. Centers for Disease Control and Prevention. Monitoring selected national HIV prevention and care objectives by using HIV surveillance data - United States and 6 dependent areas, 2014. HIV Surveillance Supplemental Report 2016;21(No. 4). Available online: http://www.cdc. gov/hiv/library/reports/surveillance/. Published July 2016. Accessed August 7th 2019.

4. Centers for Disease Control and Prevention (CDC). Trends in U.S. HIV diagnoses, 2005-2014. Available online: https://www.cdc.gov/nchhstp/newsroom/docs/ factsheets/hiv-data-trends-fact-sheet-508.pdf. Accessed August 7th 2019.

5. Centers for Disease Control and Prevention. HIV Surveillance Report, 2018; vol 31. HIV Surveillance Report: Diagnoses of HIV Infection in the United States and Dependent Areas, 2018 (updated). Available online: https://www.cdc.gov/hiv/library/reports/hiv-surveillance/ vol-31/index.html, accessed January 5th, 2020.

6. Centers for Disease Control and Prevention. HIV and Youth. Last Updated September 9, 2019. Available online: https://www.cdc.gov/hiv/group/age/youth/. Accessed October 19, 2019.

7. Chen M, Rhodes PH, Hall IH, et al. Prevalence of undiagnosed HIV infection among persons aged $>/=13$ years--National HIV Surveillance System, United States, 2005-2008. MMWR Suppl 2012;61:57-64.

8. Buchbinder SP, Liu AY. CROI 2018: Epidemic Trends and Advances in HIV Prevention. Top Antivir Med 2018;26:1-16.

9. Widman L, Choukas-Bradley S, Helms SW, et al. Adolescent Susceptibility to Peer Influence in Sexual Situations. J Adolesc Health 2016;58:323-9.

10. Kerr JC, Valois RF, Diclemente RJ, et al. HIV-related stigma among African-American youth in the Northeast and Southeast US. AIDS Behav 2014;18:1063-7.

11. Mustanski B, Morgan E, D'Aquila R, et al. Individual and Network Factors Associated With Racial Disparities in HIV Among Young Men Who Have Sex With Men: Results From the RADAR Cohort Study. J Acquir Immune Defic Syndr 2019;80:24-30.

12. Momplaisir F, Hussein M, Tobin-Fiore D, et al. Racial Inequities in HIV Prevalence and Composition of Risk Networks Among People Who Inject Drugs in HIV Prevention Trial Network 037. J Acquir Immune Defic Syndr 2017;76:394-401.

13. McNairy ML, El-Sadr WM. A paradigm shift: focus on the HIV prevention continuum. Clin Infect Dis 2014;59 Suppl 1:S12-5.

14. Bradley H, Hall HI, Wolitski RJ, et al. Vital Signs: HIV diagnosis, care, and treatment among persons living with HIV--United States, 2011. MMWR Morb Mortal Wkly Rep 2014;63:1113-7.

15. Zanoni BC, Mayer KH. The adolescent and young adult HIV cascade of care in the United States: exaggerated health disparities. AIDS Patient Care STDS 2014;28:128-35.

16. Centers for Disease C, Prevention. HIV testing among high school students--United States, 2007. MMWR Morb Mortal Wkly Rep 2009;58:665-8.

17. Caldeira KM, Singer BJ, O'Grady KE, et al. HIV testing in recent college students: prevalence and correlates. AIDS Educ Prev 2012;24:363-76.

18. Straub DM, Arrington-Sanders R, Harris DR, et al. Correlates of HIV testing history among urban youth recruited through venue-based testing in 15 US cities. Sex Transm Dis 2011;38:691-6.

19. Swenson RR, Rizzo CJ, Brown LK, et al. Prevalence and correlates of HIV testing among sexually active African American adolescents in 4 US cities. Sex Transm Dis 2009;36:584-91.

20. Kim EK, Thorpe L, Myers JE, et al. Healthcare-related correlates of recent HIV testing in New York City. Prev Med 2012;54:440-3.

21. Gagliardo C, Murray M, Saiman L, et al. Initiation of antiretroviral therapy in youth with HIV: a U.S.-based provider survey. AIDS Patient Care STDS 2013;27:498-502.

22. Giacomet V, Albano F, Starace F, et al. Adherence to antiretroviral therapy and its determinants in children with human immunodeficiency virus infection: a multicentre, national study. Acta Paediatr 2003;92:1398-402.

23. Chandwani S, Koenig LJ, Sill AM, et al. Predictors of antiretroviral medication adherence among a diverse cohort of adolescents with HIV. J Adolesc Health 2012;51:242-51.

24. Buchanan AL, Montepiedra G, Sirois PA, et al. Barriers to medication adherence in $\mathrm{HIV}$-infected children and 
youth based on self- and caregiver report. Pediatrics 2012;129:e1244-51.

25. Murphy DA, Sarr M, Durako SJ, et al. Barriers to HAART adherence among human immunodeficiency virus-infected adolescents. Arch Pediatr Adolesc Med 2003;157:249-55.

26. Martinez J, Harper G, Carleton RA, et al. The impact of stigma on medication adherence among HIV-positive adolescent and young adult females and the moderating effects of coping and satisfaction with health care. AIDS Patient Care STDS 2012;26:108-15.

27. Tanney MR, Naar-King S, MacDonnel K, et al. Depression and stigma in high-risk youth living with HIV: a multi-site study. J Pediatr Health Care 2012;26:300-5.

28. Williams PL, Storm D, Montepiedra G, et al. Predictors of adherence to antiretroviral medications in children and adolescents with HIV infection. Pediatrics 2006;118:e1745-57.

29. Gardner LI, Metsch LR, Anderson-Mahoney P, et al. Efficacy of a brief case management intervention to link recently diagnosed HIV-infected persons to care. AIDS 2005;19:423-31.

30. Israelski D, Gore-Felton C, Power R, et al. Sociodemographic characteristics associated with medical appointment adherence among HIV-seropositive patients seeking treatment in a county outpatient facility. Prev Med 2001;33:470-5.

31. Reisner SL, Jadwin-Cakmak L, White Hughto JM, et al. Characterizing the HIV Prevention and Care Continua in a Sample of Transgender Youth in the U.S. AIDS Behav 2017;21:3312-27.

32. Wheeler WH, Ziebell RA, Zabina H, et al. Prevalence of transmitted drug resistance associated mutations and HIV1 subtypes in new HIV-1 diagnoses, U.S.-2006. AIDS 2010;24:1203-12.

33. Agwu AL, Bethel J, Hightow-Weidman LB, et al. Substantial multiclass transmitted drug resistance and drug-relevant polymorphisms among treatment-naive behaviorally HIV-infected youth. AIDS Patient Care STDS 2012;26:193-6.

34. Anderson PL, Glidden DV, Liu A, et al. Emtricitabinetenofovir concentrations and pre-exposure prophylaxis efficacy in men who have sex with men. Sci Transl Med 2012;4:151ra125.

35. Marcus JL, Hurley LB, Hare CB, et al. Preexposure Prophylaxis for HIV Prevention in a Large Integrated Health Care System: Adherence, Renal Safety, and Discontinuation. J Acquir Immune Defic Syndr 2016;73:540-6.
36. Liu AY, Hessol NA, Vittinghoff E, et al. Medication adherence among men who have sex with men at risk for HIV infection in the United States: implications for preexposure prophylaxis implementation. AIDS Patient Care STDS 2014;28:622-7.

37. Raifman J, Beyrer C, Arrington-Sanders R. HIV Education and Sexual Risk Behaviors Among Young Men Who Have Sex with Men. LGBT Health 2018;5:131-8.

38. Hosek S, Celum C, Wilson CM, et al. Preventing HIV among adolescents with oral PrEP: observations and challenges in the United States and South Africa. J Int AIDS Soc 2016;19:21107.

39. Sullivan PS, Giler RM, Mouhanna F, et al. Trends in the use of oral emtricitabine/tenofovir disoproxil fumarate for pre-exposure prophylaxis against HIV infection, United States, 2012-2017. Ann Epidemiol 2018;28:833-40.

40. MacKellar DA, Valleroy LA, Secura GM, et al. Unrecognized HIV infection, risk behaviors, and perceptions of risk among young men who have sex with men: opportunities for advancing HIV prevention in the third decade of HIV/AIDS. J Acquir Immune Defic Syndr 2005;38:603-14.

41. Whitfield THF, John SA, Rendina HJ, et al. Why I Quit Pre-Exposure Prophylaxis (PrEP)? A Mixed-Method Study Exploring Reasons for PrEP Discontinuation and Potential Re-initiation Among Gay and Bisexual Men. AIDS Behav 2018;22:3566-75.

42. Mullins TL, Lally M, Zimet G, et al. Clinician attitudes toward CDC interim pre-exposure prophylaxis (PrEP) guidance and operationalizing PrEP for adolescents. AIDS Patient Care STDS 2015;29:193-203.

43. Culp L, Caucci L. State adolescent consent laws and implications for HIV pre-exposure prophylaxis. Am J Prev Med 2013;44:S119-24.

44. Hosek SG, Rudy B, Landovitz R, et al. An HIV Preexposure Prophylaxis Demonstration Project and Safety Study for Young MSM. J Acquir Immune Defic Syndr 2017;74:21-9.

45. Khanna AS, Michaels S, Skaathun B, et al. Preexposure Prophylaxis Awareness and Use in a Population-Based Sample of Young Black Men Who Have Sex With Men. JAMA Intern Med 2016;176:136-8.

46. Lelutiu-Weinberger C, Golub SA. Enhancing PrEP Access for Black and Latino Men Who Have Sex With Men. J Acquir Immune Defic Syndr 2016;73:547-55.

47. Cahill S, Taylor SW, Elsesser SA, et al. Stigma, medical mistrust, and perceived racism may affect PrEP awareness and uptake in black compared to white gay and bisexual 
men in Jackson, Mississippi and Boston, Massachusetts. AIDS Care 2017;29:1351-8.

48. Hess KM, Crawford J, Eanes A, et al. Reasons Why Young Men Who Have Sex with Men Report Not Using HIV Pre-Exposure Prophylaxis: Perceptions of Burden, Need, and Safety. AIDS Patient Care STDS 2019;33:449-54.

49. Machado DM, de Sant'Anna Carvalho AM, Riera R. Adolescent pre-exposure prophylaxis for HIV prevention: current perspectives. Adolesc Health Med Ther 2017;8:137-48.

50. Hosek SG, Landovitz RJ, Kapogiannis B, et al. Safety and Feasibility of Antiretroviral Preexposure Prophylaxis for Adolescent Men Who Have Sex With Men Aged 15 to 17 Years in the United States. JAMA Pediatr 2017;171:1063-71.

51. Celum C MN, Bekker LG, Hosek S, et al. PrEP use in young African women in HPTN 082: Effect of drug level feedback. Presented at IAS 2019. Available online: http://programme. ias2019.org/Abstract/Abstract/2328, accessed October 31, 2019.

52. Fisher CB, Arbeit MR, Dumont MS, et al. Self-Consent for HIV Prevention Research Involving Sexual and Gender Minority Youth: Reducing Barriers Through EvidenceBased Ethics. J Empir Res Hum Res Ethics 2016;11:3-14.

53. Reisner SL, Moore CS, Asquith A, et al. High risk and low uptake of pre-exposure prophylaxis to prevent HIV acquisition in a national online sample of transgender men who have sex with men in the United States. J Int AIDS Soc 2019;22:e25391.

54. Fisher CB, Fried AL, Desmond M, et al. Facilitators and Barriers to Participation in PrEP HIV Prevention Trials Involving Transgender Male and Female Adolescents and Emerging Adults. AIDS Educ Prev 2017;29:205-17.

55. Horvath KJ, Todd K, Arayasirikul S, et al. Underutilization of Pre-Exposure Prophylaxis Services Among Transgender and Nonbinary Youth: Findings from Project Moxie and TechStep. Transgend Health 2019;4:217-21.

56. Wood SM, Lee S, Barg FK, et al. Young Transgender Women's Attitudes Toward HIV Pre-exposure Prophylaxis. J Adolesc Health 2017;60:549-55.

57. Giguere R, Frasca T, Dolezal C, et al. Acceptability of Three Novel HIV Prevention Methods Among Young Male and Transgender Female Sex Workers in Puerto Rico. AIDS Behav 2016;20:2192-202.

58. Kuhns LM, Reisner SL, Mimiaga MJ, et al. Correlates of PrEP Indication in a Multi-Site Cohort of Young HIV-Uninfected Transgender Women. AIDS Behav 2016;20:1470-7.

59. Galea JT, Kinsler JJ, Salazar X, et al. Acceptability of pre- exposure prophylaxis as an HIV prevention strategy: barriers and facilitators to pre-exposure prophylaxis uptake among atrisk Peruvian populations. Int J STD AIDS 2011;22:256-62.

60. Yang D, Chariyalertsak C, Wongthanee A, et al. Acceptability of pre-exposure prophylaxis among men who have sex with men and transgender women in Northern Thailand. PLoS One 2013;8:e76650.

61. Escudero DJ, Kerr T, Wood E, et al. Acceptability of HIV Pre-exposure Prophylaxis (PREP) Among People Who Inject Drugs (PWID) in a Canadian Setting. AIDS Behav 2015;19:752-7.

62. Stein M, Thurmond P, Bailey G. Willingness to use HIV pre-exposure prophylaxis among opiate users. AIDS Behav 2014;18:1694-700.

63. Choopanya K, Martin M, Suntharasamai P, et al. Antiretroviral prophylaxis for HIV infection in injecting drug users in Bangkok, Thailand (the Bangkok Tenofovir Study): a randomised, double-blind, placebo-controlled phase 3 trial. Lancet 2013;381:2083-90.

64. Shrestha R, Copenhaver M. Exploring the Use of Preexposure Prophylaxis (PrEP) for HIV Prevention Among High-Risk People Who Use Drugs in Treatment. Front Public Health 2018;6:195.

65. Cottrell ML, Srinivas N, Kashuba AD. Pharmacokinetics of antiretrovirals in mucosal tissue. Expert Opin Drug Metab Toxicol 2015;11:893-905.

66. Patterson KB, Prince HA, Kraft E, et al. Penetration of tenofovir and emtricitabine in mucosal tissues: implications for prevention of HIV-1 transmission. Sci Transl Med 2011;3:112re4.

67. Rubtsova A, Wingood GM, Dunkle K, et al. Young adult women and correlates of potential adoption of preexposure prophylaxis (PrEP): results of a national survey. Curr HIV Res 2013;11:543-8.

68. UNAIDS. Get on the Fast-Track - The Life-Cycle Approach to HIV 2016. Available online: https://www. unaids.org/en/resources/documents/2016/get-on-the-fasttrack. Accessed October, 312019.

69. Liu A, Coleman K, Bojan K, et al. Developing a Mobile App (LYNX) to Support Linkage to HIV/Sexually Transmitted Infection Testing and Pre-Exposure Prophylaxis for Young Men Who Have Sex With Men: Protocol for a Randomized Controlled Trial. JMIR Res Protoc 2019;8:e10659.

70. Innovative Technology Network. Protocols, Available online: https://itechnetwork.org/protocols/. Accessed January 6, 2020.

71. ITECH Home Page. Available online: https:// 
itechnetwork.org/. Accessed May 8th, 2020

72. Neilan AM, Patel K, Agwu AL, et al. Model-Based Methods to Translate Adolescent Medicine Trials Network for HIV/AIDS Interventions Findings Into Policy Recommendations: Rationale and Protocol for a Modeling Core (ATN 161). JMIR Res Protoc 2019;8:e9898.

73. Hightow-Weidman LB, Muessig KE. New media challenges and opportunities. Sex Transm Infect 2017;93:309-10.

74. Hosek SG, Siberry G, Bell M, et al. The acceptability and feasibility of an HIV preexposure prophylaxis (PrEP) trial with young men who have sex with men. J Acquir Immune Defic Syndr 2013;62:447-56.

75. Mayer KH, Safren SA, Elsesser SA, et al. Optimizing PreExposure Antiretroviral Prophylaxis Adherence in Men Who Have Sex with Men: Results of a Pilot Randomized Controlled Trial of "Life-Steps for PrEP". AIDS Behav 2017;21:1350-60.

76. Biello KB, Psaros C, Krakower DS, et al. A Pre-Exposure Prophylaxis Adherence Intervention (LifeSteps) for Young Men Who Have Sex With Men: Protocol for a Pilot Randomized Controlled Trial. JMIR Res Protoc 2019;8:e10661.

77. Fjeldsoe BS, Marshall AL, Miller YD. Behavior change interventions delivered by mobile telephone short-message service. Am J Prev Med 2009;36:165-73.

78. Pop-Eleches C, Thirumurthy H, Habyarimana JP, et al. Mobile phone technologies improve adherence to antiretroviral treatment in a resource-limited setting: a randomized controlled trial of text message reminders. AIDS 2011;25:825-34.

79. Garofalo R, Kuhns LM, Hotton A, et al. A Randomized Controlled Trial of Personalized Text Message Reminders to Promote Medication Adherence Among HIV-Positive Adolescents and Young Adults. AIDS Behav 2016;20:1049-59.

80. Siegler A. Electronic Pre-Exposure Prophylaxis (PrEP) Initiation and Maintenence Home Care System (ePrEP). Clinical Trial: NCT03729570. Available online: https:// clinicaltrials.gov/ct2/show/NCT03729570?term=NCT03 729570\&rank=1, Accessed January 6, 2020.

doi: $10.21037 /$ mhealth-20-42

Cite this article as: Allan-Blitz LT, Mena LA, Mayer KH. The ongoing HIV epidemic in American youth: challenges and opportunities. mHealth 2021;7:33.
81. FDA Approves Second Drug to Prevent HIV Infection as Part of Ongoing Efforts to End the HIV Epidemic. October 2019. Available online: https://www.fda.gov/ news-events/press-announcements/fda-approves-seconddrug-prevent-hiv-infection-part-ongoing-efforts-end-hivepidemic. Accessed April 25th, 2020

82. Hare CB, Coll J, Ruane P, et al. The Phase 3 Discover Study: Daily F/TAF or F/TDF for HIV Preexposure Prophylaxis. Available online: http://www.croiconference. org/sessions/phase-3-discover-study-daily-ftaf-or-ftdfhiv-preexposure-prophylaxis. Accessed August 7th, 2019. CROI; Seattle, Washington. March 4-7, 2019.

83. Markowitz M, Frank I, Grant RM, et al. Safety and tolerability of long-acting cabotegravir injections in HIVuninfected men (ECLAIR): a multicentre, double-blind, randomised, placebo-controlled, phase $2 \mathrm{a}$ trial. Lancet HIV 2017;4:e331-e340.

84. Gunawardana M, Remedios-Chan M, Miller CS, et al. Pharmacokinetics of long-acting tenofovir alafenamide (GS-7340) subdermal implant for HIV prophylaxis. Antimicrob Agents Chemother 2015;59:3913-9.

85. Fonner VA, Dalglish SL, Kennedy CE, et al. Effectiveness and safety of oral HIV preexposure prophylaxis for all populations. AIDS 2016;30:1973-83.

86. Roland ME, Neilands TB, Krone MR, et al. Seroconversion following nonoccupational postexposure prophylaxis against HIV. Clin Infect Dis 2005;41:1507-13.

87. Nel A, van Niekerk N, Kapiga S, et al. Safety and Efficacy of a Dapivirine Vaginal Ring for HIV Prevention in Women. N Engl J Med 2016;375:2133-43.

88. Baeten JM, Palanee-Phillips T, Brown ER, et al. Use of a Vaginal Ring Containing Dapivirine for HIV-1 Prevention in Women. N Engl J Med 2016;375:2121-32.

89. Ledgerwood JE, Coates EE, Yamshchikov G, et al. Safety, pharmacokinetics and neutralization of the broadly neutralizing HIV-1 human monoclonal antibody VRC01 in healthy adults. Clin Exp Immunol 2015;182:289-301.

90. Hua CK, Ackerman ME. Increasing the Clinical Potential and Applications of Anti-HIV Antibodies. Front Immunol 2017;8:1655. 